\title{
Ultrasound Frequency Analysis of a Magnetic Fluid in Low-Intensity External Magnetic Field
}

\author{
J. KurimskÝ ${ }^{a, *}$, M. RajñÁK ${ }^{a, b}$, R. Cimbala $^{a}$, B. Dolník ${ }^{a}$, J. Tóthová $^{a}$, K. PauloviČová $^{b}$, \\ M. Timko ${ }^{b}$, P. KopČAnskÝ ${ }^{b}$, I. Kolcunová ${ }^{a}$, J.Â Petrás $\check{S}^{a}$, J. DŽMura ${ }^{a}$ And J. Balogh ${ }^{a}$ \\ ${ }^{a}$ Faculty of Electrical Engineering and Informatics, Technical University of Košice, Letná 9, 04154 Košice, Slovakia \\ ${ }^{b}$ Institute of Experimental Physics, SAS, Watsonova 47, 04001 Košice, Slovakia
}

\begin{abstract}
This work deals with an interaction of a magnetic fluid of a dielectric nature with a magnetic field by means of ultrasound waves measurements and analysis. Ultrasound analysis is known as a non-destructive inspection tool often used in technical diagnostics, moreover, it has numerous applications in medicine and biology, too. We report the low-frequency ultrasound analysis of a dielectric magnetic fluid in a low-intensity external static magnetic field. The studied magnetic fluid was composed of a transformer oil and dispersed magnetite nanoparticles coated with oleic acid. Experiments were carried out by using an ultrasonic testing cell. The cell was exposed to a magnetic field of $50 \mathrm{mT}$ in both parallel and perpendicular direction to the waves propagation. A through-transmission mode measurement was applied, where two fixed narrow-band transducers with completely shielded crystal for maximum RFI/EMI immunity (Physical Acoustic R15I-AST, the resonant frequency $150 \mathrm{kHz}$ ) served as a transmitter and a receiver. In this way we carried out the measurement of the frequency-dependent ultrasonic response to a rectangle calibrating signal of $5 \mu$ s pulse width. Digitized signals were recorded for further analysis. We present the frequency domain analysis of the low-frequency ultrasound in magnetic fluid. The frequency spectrum in magnetic fluid colloidal system was calculated by the Fourier transformation method. Results show that there is a frequency shift in the amplitude-frequency spectrum caused by the step-up magnetic field. The higher the magnetic field, the higher the frequency of the peaks. The effect of particle aggregation in magnetic field on the ultrasound wave propagation is discussed in the paper.
\end{abstract}

DOI: 10.12693/APhysPolA.131.910

PACS/topics: 43.58.+z, 43.60.+d, 47.65.Cb, 82.70.Dd, 83.80.Hj

\section{Introduction}

To provide a material characterization and/or several spatio-temporal phenomena examinations and make measurements, the power of ultrasonic waves is used often. The general advantage of such approach is that prior modifications of the sample under test is not required. Over the last decade an increasing number of publications can be identified with the topic of ultrasound in magnetic fluids (MF). There are several theoretical and experimental approaches developed recently to investigate ultrasonic characterization of colloidal solutions, e.g. [1] and polar or non-polar carrier-based MF, e.g. [2, 3]. Both fluid matters are of a specific features moderated by solid particles at certain concentrations.

The transformer oil-based MF is a colloidal solution of dielectric oil and magnetite nanoparticles (NP). The recent works were motivated by possibility to improve the dielectric and thermal properties of high performance insulating fluids and the knowledge transfer to modern power technologies. Regarding ultrasonics in MFs, one can find studies on external thermal and magnetic field influence on MF structure [4], the particle size distribution and aggregates in MF $[5,6]$ and basic sonic characterization of MFs, too [7, 8].

\footnotetext{
*corresponding author; e-mail: juraj.kurimsky@tuke.sk
}

In this work a special attention is given to the attributing of the ultrasonic waves in MFs to the applicable nondestructive testing (NDT). That is why we select a relatively narrow band of the frequency spectra which is applicable and commonly used for NDT in power transformers. Herein referenced works deal with the ultrasonic frequencies above the megahertz, while our approach is based on the lower frequencies used in the current inspection tools. The ultrasonic measurements have found an important application in partial discharge (PD) inspection $[9,10]$. It is known that PDs are dangerous and they accelerate the development of the breakdown process. Such PDs are generated in the places of weakened insulating ability inside the oil-immersed transformers and generate an acoustic disturbance. There are known methods for localization of such PD sources by means of acoustic wave measurement [11, 12]. The question is, what happens to acoustic waves when transformer insulating fluid becomes the MF? Consequently, it is necessary to investigate the acoustic response in both fluids within PD inspection frequency range. This is the first investigation on frequency spectra change in MF in the kilohertz domain. We present also the differences between sonic responses of a transformer oil and MFs in a low-intensity external magnetic field.

\section{Experimental}

The experiments were performed in the MF composed of transformer oil and $\mathrm{Fe}_{3} \mathrm{O}_{4}$ NPs. NPs were obtained 
by chemical precipitation and stabilized by oleic acid $\mathrm{C}_{18} \mathrm{H}_{34} \mathrm{O}_{2}$ in the carrier fluid [13]. The mean magneticcore diameter $d_{h}$ was $33.28 \mathrm{~nm}$. The list of the final material characterization, including magnetization measurements, follows: the material density $\rho$, the dynamic viscosity $\eta$, the saturation magnetization $M_{s}$, dc magnetic susceptibility $\chi_{d c}$, the magnetic volume fraction $\Phi_{V}$, the magnetic mass fraction $\Phi_{m}$, refer to Table I.

TABLE I

Magnetic and rheological parameters of fluid samples at $20^{\circ} \mathrm{C}$.

\begin{tabular}{c|c|c|c|c|c|c}
\hline \hline Sample & $\begin{array}{c}\rho \\
{\left[\frac{\mathrm{g}}{\mathrm{cm}^{3}}\right]}\end{array}$ & $\begin{array}{c}\eta \\
{[\mathrm{mPa} \mathrm{s}]}\end{array}$ & $\begin{array}{c}M_{s} \\
{\left[\frac{\mathrm{A}}{\mathrm{m}}\right]}\end{array}$ & $\begin{array}{c}\chi_{d c} \\
{[-]}\end{array}$ & $\begin{array}{c}\Phi_{V} \\
{[\%]}\end{array}$ & $\begin{array}{c}\Phi_{m} \\
{[\%]}\end{array}$ \\
\hline oil & 0.84 & 14.59 & - & - & - & - \\
MF_M & 1.28 & 53 & 1280 & 0.765 & 6.6 & 24.1
\end{tabular}

In the ultrasonic measurements the narrow-band transducers (Physical Acoustic) were used with the resonant frequency of $150 \mathrm{kHz}$. Two transducers, one acting as a transmitter and the other as a receiver, were operated in a through-transmission mode measurement. They were fixed at the ends of the tube-shaped test cell. Designed material of the tube was aluminum with the thickness of $1 \mathrm{~mm}$. The cell dimensions are as follows: the length of $100 \mathrm{~mm}$, the inner diameter of $28 \mathrm{~mm}$.

In order to analyze the effect of the magnetic field on the frequency response in MF, the test cell was placed between a pair of coils in the Helmholtz arrangement. It was calibrated to generate field up to $50 \mathrm{mT}$. The ultrasonic measurements under magnetic field were carried out for two values of the angle $\gamma$ between the direction of wave propagation and the magnetic field $B: 0^{\circ}$ and $90^{\circ}$, see Fig. 1a,b. The measurements were done at ambient conditions: a temperature of $23.5^{\circ} \mathrm{C}$, a relative humidity of $65 \%$. In this way we carried out the measurements of the frequency-dependent ultrasonic response to a burst initiated by a rectangle calibrating signal of $5 \mu$ s pulse width at amplitude of $-25 \mathrm{~V}$, partially seen in Fig. 1c. Digitized signals were recorded for a further analysis.
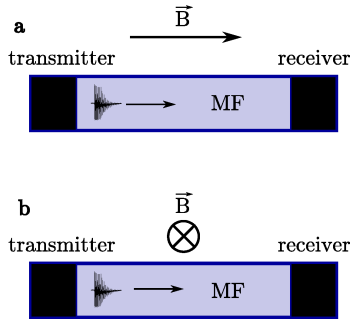

Fig. 1. Mutual direction of the wave propagation and the magnetic field $(\mathrm{a}, \mathrm{b})$, acoustic response in MF (c).

\section{Results and discussion}

Spectral analysis of the signals and the peak detection were implemented in the Scilab. The number of dominant frequencies (DF) is the same for pure oil and MF, too. However, the frequency peaks of MF response are shifted down over $50 \mathrm{kHz}$, see Fig. 2. Eight DFs for both samples can be identified.

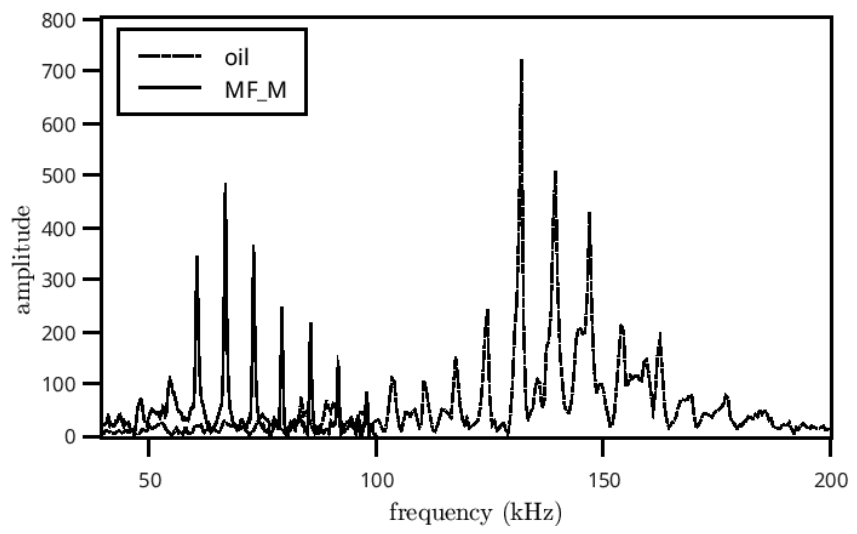

Fig. 2. The change of the most dominant peak frequency due to the magnetic field in the MF sample.

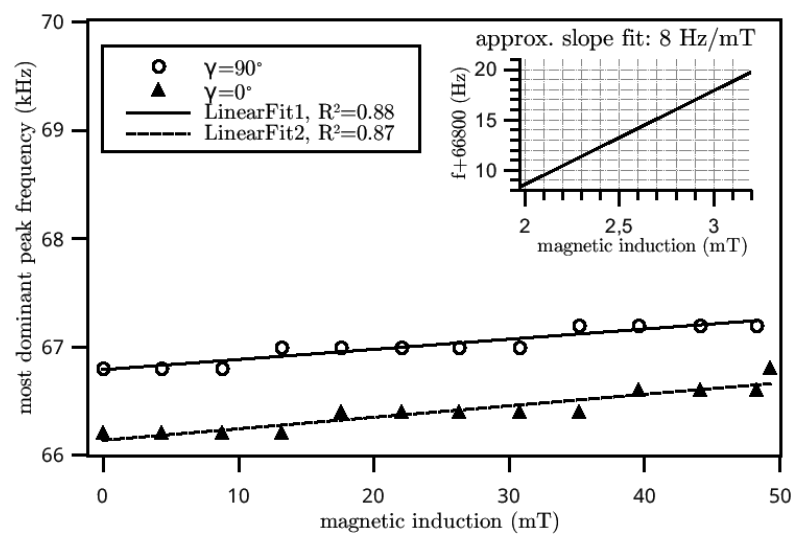

Fig. 3. The change of the most dominant peak frequency due to the magnetic field in the MF sample.

The application of the magnetic field influences the DFs peak in MF. The measurements showed that the peaks tend to shift to higher frequencies with increase of magnetic field. In order to make a clear presentation of the state, the increase for the most (M)DF due to magnetic field is shown, see Fig. 3. In the figure the lines represent linear fits of the experimental data. The increase of MDF per $1 \mathrm{mT}$ is shown in the inset. At given experimental conditions the increase of approximately $8 \mathrm{~Hz} / \mathrm{mT}$ can be derived for DFs in the used MF sample. However, in the pure oil, the peaks of the MDF remained stable for a whole range of applied magnetic field $(131.5 \mathrm{kHz})$. The physical properties of MF are moderated by the influence of the external magnetic field. The MF sample volume at the thermal equilibrium interacts with the applied magnetic field, resulting in the NPs alignment in the field direction. The formation of NP aggregates takes place. Certainly, they are of a bigger diameter than the raw NPs, so the friction factor rises. Subsequently, it leads to 


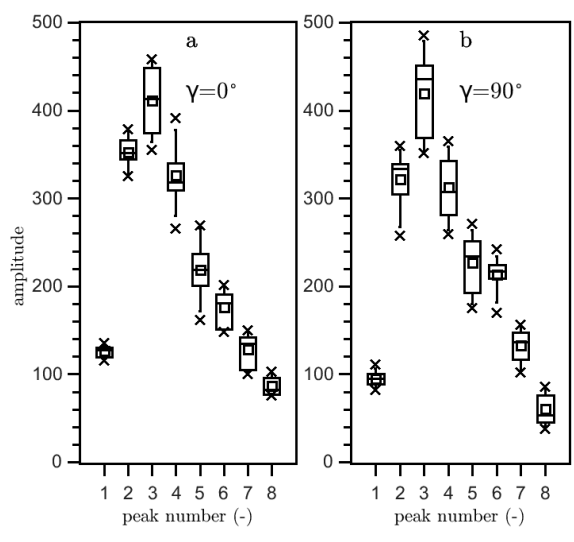

Fig. 4. Box statistics on dominant frequency amplitude variations, parallel (a) and perpendicular (b) configuration.

a rise of the ultrasonic attenuation. On the other hand, new reconfiguration of immersed particle size, due to aggregating process, causes the ultrasonic properties of the MF to resemble those of the basic carrier.

Although the anisotropy of ultrasonic velocity has been reported, e.g. [14], for the investigated cases we observed the same increase of MDFs in the given range of magnetic field. This is shown in Fig. 4 in the form of box-plot statistics. The DF peak amplitude data, as measured in the given range of magnetic field, do not indicate any significant concurrent variations.

\section{Conclusions}

The study reveals the differences between the frequency spectra of ultrasonic waves generated in pure transformer oil and MF. The effect of the frequency spectra variation due to the low magnetic field has been confirmed in the MF. It has been shown that MDF peak yields the slope $8 \mathrm{~Hz} / \mathrm{mT}$ approximately. At experimental conditions, the anisotropy of that feature has not been observed. The information is useful for design of current power transformer NDT inspection systems capable of PD detection which utilize the information extracted from the change of ultrasonic waveforms generated by PD sources. The feature can be well adopted by data processing procedures.

\section{Acknowledgments}

The work was supported by: Slovak Academy of Sciences and Ministry of Education in the framework of Projects VEGA No. 1/0132/15, 1/0311/15, and 2/0141/16; Ministry of Education Agency for structural funds of EU in frame of Projects No. 26220120003, 6220120046, and 26220120055; Slovak Research and Development Agency project No. APVV-15-0438.

\section{References}

[1] P.V. Lebedev-Stepanov, S.A. Rybak, Acoust. Phys. 57, 801 (2011).

[2] M. Nabeel Rashin, J. Hemalatha, Ultrasonics 52 , 1024 (2012).

[3] K. Parekh, J. Patel, R.V. Upadhyay, Ultrasonics 60, 126 (2015).

[4] J. Kudelcik, P. Bury, J. Drga, P. Kopcansky, V. Zavisova, M. Timko, J. Magn. Magn. Mater. 326, 75 (2013).

[5] A. Jozefczak, T. Hornowski, A. Skumiel, Int. J. Thermophys. 32, 795 (2010).

[6] R. Badescu, G. Apreotesei, V. Badescu, Rom. Rep. Phys. 55, 173 (2010).

[7] D.Y. Chung, W.E. Isler, J. Appl. Phys. 49, 1809 (1978).

[8] A. Jozefczak, J. Magn. Magn. Mater. 256, 267 (2003).

[9] T. Boczar, D. Zmarzly, IEEE T. Dielect. El. In. 11, 433 (2004).

[10] J. Petras, J. Kurimsky, J. Balogh, R. Cimbala, J. Dzmura, B. Dolnik, I. Kolcunova, Acta Acust. United Acust. 102, 16 (2016).

[11] M.S. Naderi, T.R. Blackburn, B.T. Phung, M.S. Naderi, A. Nasiri, Electr. Pow. Syst. Res. 78, 202 (2008).

[12] H.-L. Liu, Appl. Acoust. 102, 71 (2016).

[13] M. Rajnak, J. Kurimsky, B. Dolnik, K. Marton, L. Tomco, A. Taculescu, L. Vekas, J. Kovac, I. Vavra, J. Tothova, P. Kopcansky, M. Timko, J. Appl. Phys. 114, 34313 (2013).

[14] T. Hornowski, A. Jozefczak, B. Kolodziejczyk, M. Timko, A. Skumiel, M. Rajnak, J. Phys. D Appl. Phys. 48, 175303 (2015). 